\title{
A Holistic Decision Support Environment for Web Accessibility
}

\author{
Yehya Mohamad $\left.^{1(}\right)$, Carlos A. Velasco ${ }^{1}$, Nikolaos Kaklanis ${ }^{2}$, \\ Dimitrios Tzovaras ${ }^{2}$, and Fabio Paterno ${ }^{3}$ \\ ${ }^{1}$ Fraunhofer Institute for Applied Information Technology (FIT), \\ Schloss Birlinghoven, 53757 Sankt Augustin, Germany \\ \{Yehya.mohamad, Carlos.velasco\} @efit. fraunhofer.de \\ 2 Information Technologies Institute, Centre for Research and Technology Hellas, \\ 6th Klm. Charilaou-Thermi Road, P.O. BOX 60361, 57001 Thessaloniki, Greece \\ \{nkak, Dimitrios. Tzovaras\}@iti.gr \\ ${ }^{3}$ CNR-ISTI, HIIS Laboratory, Via Moruzzi 1, 56124 Pisa, Italy
}

\begin{abstract}
This paper presents the architecture of a decision support environment for large-scale assessment of compliance against web accessibility recommendations and legislations. The proposed decision support environment aims at integrating, extending and further enhancing existing web accessibility solutions making them customizable to the needs of different stakeholders, transferable to different sectors in web and mobile environments, in order to minimize costs and development time by also increasing scalability and improving their accessibility and usability.
\end{abstract}

Keywords: Web accessibility · Decision support system Accessibility guidelines

\section{Introduction}

It is important to realise that people with disabilities are not just a tiny minority of the population of the European Union. The lowest estimate, based on currently defined impairment categories, indicates their total number at around 44 Million persons (nearly $13 \%$ of the population of the EU). Designing for people with disabilities is becoming an increasingly important topic for a variety of reasons, especially due to the recent legislation (European Web Accessibility Directive, WAD ${ }^{1}$ ) published on 2 December 2016 and entered into force on 22 December 2016 in Europe promoting the rights of disabled people. In this direction, accessibility has become necessary due to the rapid growth of online information and interactive services provided by web and mobile applications. Some examples are online banking and shopping, public services, social networks. The lack or absence of accessibility contributes to the exclusion or partial exclusion of many people from society.

\footnotetext{
${ }^{1}$ http://data.europa.eu/eli/dir/2016/2102/oj .
} 
Making web and mobile apps more accessible results in a better user experience for all, not just for users with disabilities. Simple changes that make applications more userfriendly can bring huge improvements for everyone. Accessibility also benefits businesses with economic gains, as they can reach a larger customer base when it is supported. Web accessibility is not just about technical standards, web architecture and design. It is an issue in terms of political will and moral obligation, now enshrined in the United Nations Convention on the Rights of Persons with Disabilities (UNCRPD). Article 9 of the Convention, to which the EU is a party, requires that appropriate measures are taken to ensure access for persons with disabilities, on equal basis with others, to inter alia information and communication technologies, including the Internet. By that, people with disabilities will have better access to the websites and mobile applications of public services. Web Accessibility Evaluation is an assessment procedure to analyse how well the Web can be used by people with different levels of disabilities. However, there are two main categories of web testing tools: - Tools for accessibility testing and repair, like Siteimprove, AccVerify, imergo® [7], WaaT [6], egovMon, AChecker, SortSite, TAW, Deque, MAUVE etc. The metrics implemented by these tools correspond (more or less accurately) to official (W3C WAI or Section 508) accessibility criteria. - Tools for usability testing (e.g. WebXM, LIFT, WebCriteria) that are based on usability guidelines. A comprehensive list of such tools can be found on $\mathrm{W} 3 \mathrm{C} /$ WAI website ${ }^{2}$. Having an automated way of evaluating the accessibility of Web pages opens the way to perform large-scale analysis of Web accessibility. Large-scale accessibility evaluations of the Web are not yet well established. This may be due to the dependency of computational resources for large-scale analysis [1]. Current assessment tools had a number of limitations: First, most of these tools allow validating and or evaluating only single webpages, or in rare cases, single websites [5]. However, in the real world it is more useful to evaluate the accessibility of collections of websites grouped by topic or territory and to monitor the evolution of their accessibility over time, providing a high-level view of whether progress is being made. To mitigate this problem, work is typically conducted on evaluating smaller scale collections of Web documents [2]. These processes are based on sampling methods, such as those defined in UWEM [3]. However, there is always a significant sampling bias induced by these methods [4]. In this paper, the general design and architecture of a decision support environment for large scale web accessibility assessment are presented.

\section{Architecture of the Proposed Infrastructure}

In the current situation introduced before, a holistic and hybrid approach to Web accessibility assessment is still missing. This approach will provide developers, designers, experts and policy makers with an integrated web accessibility support environment. This will be obtained with a dynamic and personalised environment that will enable them, on one hand, to design accessible software applications and, on the other hand, to understand their problems, and analyse and test their accessibility. Within this approach, the challenge is to find the way in which technology can be best used to increase

\footnotetext{
${ }^{2}$ https://www.w3.org/WAI/ER/tools/ .
} 
accessibility, usability and quality of software applications and services. The presented architecture integrates both new ICT driven concepts and user-oriented approaches with methodologies and tools regarding web accessibility assessment. Within this context, and by using lessons learnt from relevant research and best practices (WCAG, ARIA, EARL, etc.), this architecture aims at developing a new scalable, interoperable and integrated web accessibility assessment and Decision Support Environment (DSE) system as an accessible-driven solution with a user-centred approach. Instead of a simple developer and designer-aid framework, the designer/developer communities will have a usercentred environment to get access to the different parts of the methodological approaches they need. The resulting instances of this architecture will be installable in several ways e.g. as standalone environment, software as a service ( $\mathrm{SaaS})$, platform as a service (PaaS) or just as an API for integration with CMSs or IDEs (See Fig. 1.).

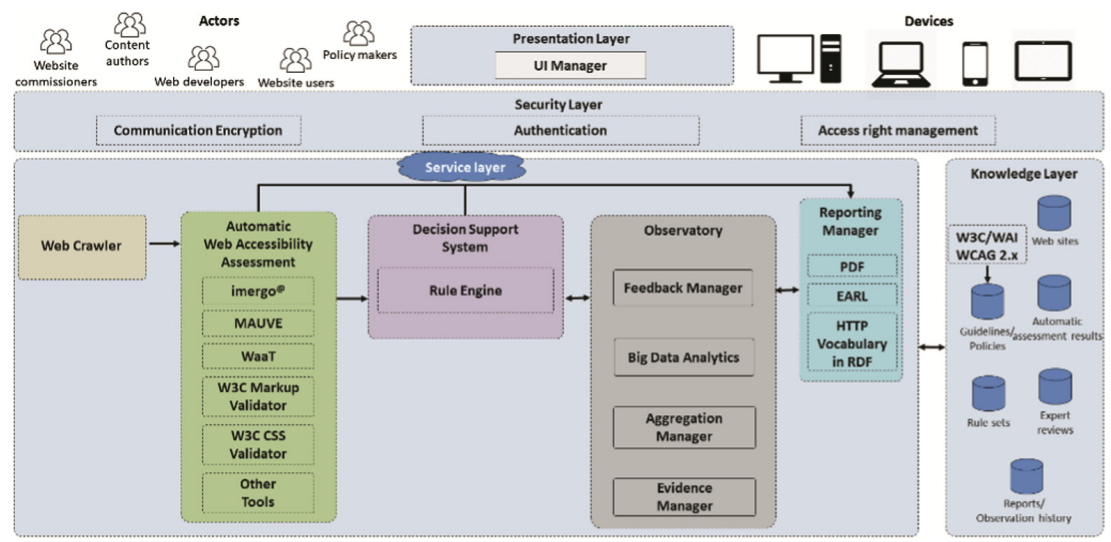

Fig. 1. Architecture of the proposed infrastructure

The core components of the software platform can be split into 4 logical layers: (1) The knowledge layer that includes all the knowledge/data needed for the proper functioning of the large scale components (e.g. list of web sites to be evaluated, guidelines and policies to be considered in web accessibility assessment, rule sets, etc.) as well as the knowledge/results that will be extracted (e.g. ontologies ${ }^{3}$ that describe the guidelines of WCAG 2.x and ARIA standards in a semantic manner, etc.). (2) The service layer that includes all the core software modules of the large scale cloud-based architecture. In this layer we include our accessibility validation tools (Imergo, MAUVE, Waat), tools for validating the Web sites content, and other relevant tools. (3) The security layer that is responsible for the security, authentication and access rights management. (4) The presentation layer, which includes all the user interfaces that enable user interaction with the large-scale services. We aim to provide adaptive ways to report the detected accessibility issues, also depending on the target communities (developers, end users, web site commissioners, etc.).

\footnotetext{
${ }^{3}$ http://www.accessible-eu.org/index.php/ontology.html .
} 


\section{Conclusions and Future Work}

This paper presents the high-level design of a large scale infrastructure that offers the following functionalities: (1) Automatic web accessibility assessment. (2) Decision support to accessibility experts for detecting further barriers that need further manual investigation. (3) guidance to developers in repairing accessibility errors, (4) presentation of aggregated results through an Observatory using advanced visual analytics methods enabling different stakeholders such as web commissioners, policy makers and the general public to examine changes in the accessibility of various websites. The proposed infrastructure provides also APIs to external tools like CMSs for checking webpages during design and content authoring to make them "born accessible".

We also plan to empirically validate the results of such accessibility infrastructures with the relevant communities.

Acknowledgments. This work was partially funded by the European Commission H2020 programme; Contract number 780206; WADcher. The authors would like to acknowledge the support of the WADcher consortium.

\section{References}

1. Lopes, R., Gomes, D., Carriço, L.: Web not for all: a large scale study of web accessibility. In: Proceedings of the 2010 International Cross Disciplinary Conference on Web Accessibility (W4A), p. 10. ACM, April 2010

2. Mirri, S., Muratori, L.A., Roccetti, M., Salomoni, P.: Metrics for accessibility on the Vamolà project. In: Proceedings of the 2009 International Cross-Disciplinary Conference on Web Accessibility (W4A), pp. 142-145. ACM, April 2009

3. Bühler, C., Heck, H., Perlick, O., Nietzio, A., Ulltveit-Moe, N.: Interpreting results from large scale automatic evaluation of web accessibility. In: Miesenberger, K., Klaus, J., Zagler, Wolfgang L., Karshmer, Arthur I. (eds.) ICCHP 2006. LNCS, vol. 4061, pp. 184-191. Springer, Heidelberg (2006). https://doi.org/10.1007/11788713_28

4. Brajnik, G., Mulas, A., Pitton, C.: Effects of sampling methods on web accessibility evaluations. In: Proceedings of the 9th International ACM SIGACCESS Conference on Computers and Accessibility, pp. 59-66. ACM, October 2007

5. Paternò, F., Schiavone, A.G.: The role of tool support in public policies and accessibility. ACM Interact. 22(3), 60-63 (2015)

6. Oikonomou, T., Kaklanis, N., Votis, K., Kastori, G.E., Partarakis, N., Tzovaras, D.: Waat: personalised web accessibility evaluation tool. In: Proceedings of the International CrossDisciplinary Conference on Web Accessibility, p. 19. ACM (2011)

7. Carlos, V., Denev, D., Stegemann, D., Mohamad, Y.: A web compliance engineering framework to support the development of accessible rich internet applications. ACM. https:// doi.org/10.1145/1368044.1368054, 978-1-60558-153-8 
Open Access This chapter is licensed under the terms of the Creative Commons Attribution 4.0 International License (http://creativecommons.org/licenses/by/4.0/), which permits use, sharing, adaptation, distribution and reproduction in any medium or format, as long as you give appropriate credit to the original author(s) and the source, provide a link to the Creative Commons license and indicate if changes were made.

The images or other third party material in this chapter are included in the chapter's Creative Commons license, unless indicated otherwise in a credit line to the material. If material is not included in the chapter's Creative Commons license and your intended use is not permitted by statutory regulation or exceeds the permitted use, you will need to obtain permission directly from the copyright holder. 